Portland State University

PDXScholar

2014

\title{
Cultural Competency: a Quantitative Analysis of Cultural Awareness in U.S. Healthcare
}

Jordan Nomie

Portland State University

Follow this and additional works at: https://pdxscholar.library.pdx.edu/honorstheses

Let us know how access to this document benefits you.

\section{Recommended Citation}

Nomie, Jordan, "Cultural Competency: a Quantitative Analysis of Cultural Awareness in U.S. Healthcare" (2014). University Honors Theses. Paper 65.

https://doi.org/10.15760/honors.49

This Thesis is brought to you for free and open access. It has been accepted for inclusion in University Honors Theses by an authorized administrator of PDXScholar. Please contact us if we can make this document more accessible: pdxscholar@pdx.edu. 
Cultural Competency: A Quantitative Analysis of Cultural Awareness in U.S. Healthcare By

Jordan Nomie

An undergraduate honors thesis submitted in partial fulfillment of the requirements for the degree of

Bachelor of Science

in

University Honors

and

General Science

Thesis Advisor

Dr. Edward Kahl

Portland State University

2014

Nomie | 1 


\begin{abstract}
A growing issue in healthcare in the United States is whether consideration and attentiveness of physicians and other healthcare providers towards a patient's culture has an effect on the treatment process. An anthropological term, cultural competency, describes a medical professional's respect and integration of the patients' cultural beliefs and practices throughout the treatment process in order to reduce cross-cultural health disparities and to improve overall satisfaction. Although there is significant literature outlining a theoretical need for increased implementation of these practices, little work has been done to address actual quantitative evidence supporting or refuting the articulated need. The goal of this project is to collect and synthesize some previous quantitative findings with my own data analysis from the U.S. Department of Health and Human Services records, and integrate those findings with some of the prominent qualitative work that has framed the discourse in order to establish or refute a need for more culturally competent practices in U.S. healthcare. After analyzing economic aspects and implications, changing demographic information, and trends in treatment services; I argue there is an increasing need to implement culturally competent evaluation and practices. However, as the data suggests, further work must be done to identify specific areas of impact and the role differing cultural dynamics play so that treatment services can be modified accordingly.
\end{abstract}




\section{Table of Contents}

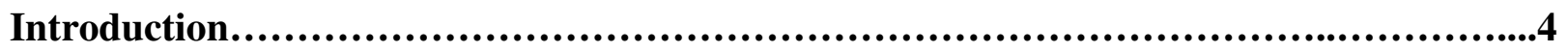

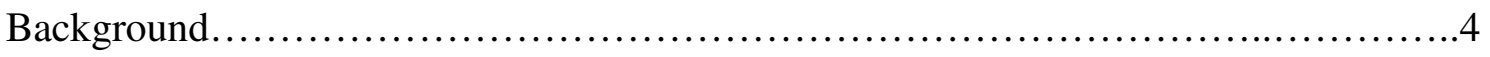

The Purnell Model........................................................... 10

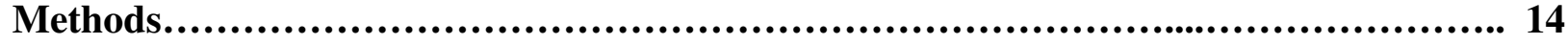

Quantitative information generated from outside sources....................... 14

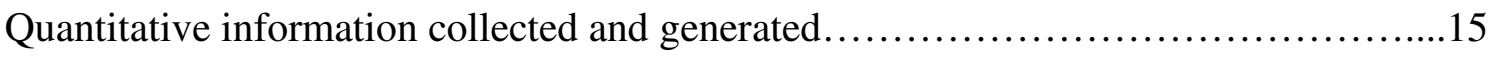

Results/ Findings.......................................................................18

Figures $2 \& 3:$ Institutional costs and benefits................................... 19

Figure 4: U.S. Demographic Changes.........................................21

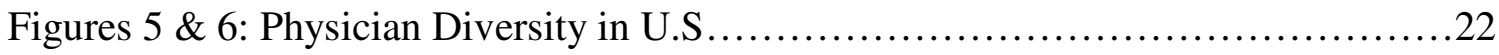

Figure 7: The impact of diversity on patient treatment.......................... 24

Conclusions/ Discussion.............................................................. 24

References.................................................................................. 28 


\section{Introduction}

\section{Background:}

I am a premedical undergraduate student, with an anticipated major in General Science and minor in Anthropology. I have always been interested in the intersection of my areas of study, more importantly how the empirical nature of science operates within the framework of actual human interactions. My interest in medicine, ability to interpret scientific data, and understanding of anthropological theory make a quantitative analysis of cultural competency my ideal area of study. Cultural competency is an anthropological term describing health care professionals' level of consideration of a patient's culture during the diagnosis and treatment of illness. Medicine has long been categorized with the sciences because it utilizes the scientific method in the establishment of diagnostic and treatment techniques. Historically, it has been perceived that the scientific basis for medical knowledge meant that the treatment of patients could also be conducted in an empirical manner (Nordqvist, 2012). A heart is a heart, regardless of the human being it resides in. All human body systems operate under the same physiological principles, unless a specific medical disorder is disrupting a normal process. However, are we all really the same? People are a product of the unique social and cultural circumstances they have been exposed to, and these unique circumstances may influence the receptiveness and effectiveness of healthcare treatment. Little work has been done to show the costs and benefits of culturally competent practices.

It is important to note that with respect to data and terminology correlation, cultural differences are assumed to be encompassed by what is reported by the various data sources as racial/ ethnic difference. Although there are innumerable cultural differences within these 
fictional "race" categories and within ethnic origins, they are simply the way differences are documented. With that being said, differences between these classifications of people very much represent cultural differences between individuals. For instance, it cannot be definitively stated that culturally competent practices need to be aimed toward a particular racial/ ethnic group because the level of diversity within those groups is immense. What can be stated is that the diversity between the groups themselves encompasses diversity in culture. Identification within particular racial/ethnic groups inherently involves identification of cultural difference. Even though there may be some level of error associated, it is the only means to use sufficient health statistics to address the issue of cultural competency in U.S. healthcare.

In order to understand cultural competency, it is important to first outline what "culture" means in the context of this work. Culture will be defined as, "the totality of socially transmitted behavioral patterns, values, customs, lifeways, arts, and all other products of human work and thought characteristics of a population of people that guide their world view and decision making" (Purnell, 2011, p.30). Cultural competency therefore is a medical professional's respect and integration of the patients' cultural beliefs and practices throughout the treatment process in order to reduce cross-cultural health disparities and to improve overall satisfaction. Crosscultural health disparities between minorities and non-minorities with respect to disease and mortality are prevalent throughout the United States (Hancock, 2005, p.5). To date, the major response by health hospitals and medical centers is an attempt at increasing the number of health providers that are representative of these minority groups, which include African Americans, Hispanics, Native Americans, and Asians. However, there has been a failure by these institutions to teach health professionals evaluation approaches and methods to address cultural diversity with respect to their patients (Hopson, 2003, p.1). Even with ever increasing cultural diversity in 
American communities, attention to multicultural and culturally competent practices in medicine have not drawn significant support. The need for more culturally competent medical practices is being articulated by many academic disciplines; however actual practices in the medical field do not reflect this. This thesis will build off previous theoretical and qualitative work to establish a basis and foundation for the concepts of cultural competency. Through my research, I have found a significant lack of quantitative analysis of culturally competent practices, namely the costs and benefits. This work will synthesize the qualitative information with statistical data, in order to analyze cultural competency in the United States more holistically. Are there tangible costs and benefits associated with culturally competent practices in the medical field, and does one outweigh the other? "The large claims about the value of cultural competence for the art of professional care-giving around the world are simply not supported by robust research showing that systematic attention to culture really improves clinical services" (Kleinman \& Benson, 2006). Perhaps articulating real manifestations of culturally competent practices or a lack thereof, will help push for the implementation of training or development programs that will formally instruct health professionals on cultural evaluation.

This issue contains important social, political, and economic elements that could have implications on how health care is administered and received in the United States. Perhaps the most important social implication is related to the current and projected changes to demographics in the United States. "Currently, the US foreign-born population comprises a larger segment than at any time in the past five decades. This trend is expected to continue" (Georgetown University, n.d.). With growing diversity in United States communities, it will become increasingly important for hospitals and medical centers to adopt culturally competent practices in order to efficiently provide effective healthcare. The increasing need for implementation of these 
practices in response to changing demographics is also important in eliminating health disparities across diverse cultural groups. Although there have been major advances in healthcare nationally, there remains significant disparities in death and illness in several cultural minority groups, as compared to the United States as a whole.

The need for more culturally competent practices also has important political implications. Hospitals and medical centers have multiple responsibilities to deliver culturally competent healthcare based on federal, state, and local regulations. There are federal civil rights laws and state and local bureaus meant to ensure equal treatment and participation in healthcare services for individuals on the basis of race, national origin, etc. The issue with most of the current legislature, accreditation, and regulatory mandates is that they fail to address areas such as: language, gender, socioeconomic status, housing status and regional differences with respect to culture (Georgetown University, n.d.). There are federal and state agencies that set and monitor standards requiring cultural competence in healthcare, and this work may be able to provide insight into developing more complete, culturally specific standards by which hospitals and medical centers will need to comply.

The issue of providing culturally competent care also has possible economic impacts on both the healthcare providers and institutions as a whole. Marketing healthcare services and delivering cost-effective healthcare are important issues for hospitals and medical centers. In order to diversify recruitment and increase patient satisfaction, "managed care plans must incorporate culturally competent policies, structures and practices to provide services for people from diverse ethnic, racial, cultural and linguistic backgrounds" (Georgetown University, n.d.). There are also important liability concerns for healthcare providers. In some hospitals and medical centers, physicians have the right to refuse to treat a patient if the patient's cultural or 
religious beliefs reduce the chance of successful treatment. For instance, Jehovah's Witnesses do not believe in receiving blood transfusions, which may be required to save the life of the patient in surgery. Lack of cultural competence may increase the chance of malpractice because of a failure of awareness. Some examples of issues that may arise from a lack of cultural competence are an absence of informed consent, breaches of professional standards of care, and accusations of negligence. Aside from criminal implications, these issues related to liability and malpractice claims have important financial and psychological impacts on the careers of providers.

Historically, there have been two major strands of discourse pertaining to cultural competency, which vary based on the levels of healthcare they address. The first strand focuses on cultural evaluator understanding through an international and cross-cultural context. This deals with how the United States, or other Western countries, understands when and how culture matters when working with other countries. In 1985, Michael Patton's New Directions on Program Evaluation proposed the question, "What happens when we export the ideas, concepts, models, methods, and values of evaluation to other countries and cultures?" (Hopson, 2003, p.3). Patton's work would become the cornerstone for those working with cultural competency in an international setting. His work is considered to be arguably the most influential in the field and by addressing the question he proposed, was able to provoke Western perspectives to consider international, cross-cultural evaluation.

The second strand of discourse focuses on cultural evaluator skill, institutional capacities, and other issues through the lens of multicultural diversity in the United States. There are no individuals significantly important to this field; rather it has been shaped by contributions from interdisciplinary work. These disciplines include but are not limited to: educational research, human development, and ethnography. Perhaps the most influential discipline in dealing with 
domestic issues of cultural competence is psychology (Hopson, 2003, p.6). Cultural competency at the level of the United States is one of the most discussed terms in psychology because of its implications on the practitioners' relationships with potentially culturally diverse patients. In psychology, cultural competence is trending toward field evaluation with the influence of AnnaMarie Madison's work, New Directions for Program Evaluations. She looked to understand the methods of diversity program planning and design among culturally diverse groups, for instance, by observing how these groups identify social problems relevant to them. This is an example of how cultural competency within the United States is being addressed in psychology, and although the aim and method varies for other disciplines, the scale, issues, and even insight are similar. It is important to note that rarely do scholars incorporate both the international, crosscultural and domestic, multicultural approaches in a single work. This work is no different, as I will be dealing almost entirely with the domestic, multicultural aspect perhaps addressing some international implications in a minor way as a launching point for future work.

Perhaps the most widely studied and arguably most influential model was outlined by Larry Purnell, which he called The Purnell Model for Cultural Competence. Not only does it explicitly outline ideas of what defines culture and how cultural competence should be understood, he provides diagrams, charts, and lists that provide a breadth and depth of framing cultural competence unlike any other model (Purnell, 2011). There are a seemingly infinite number of example questions and situations, which make the model like an encyclopedia for healthcare providers to reference when dealing with culturally diverse patients. 


\section{The Purnell Model:}

The formulation of this work involves the intersection of data from three methodological operations. The qualitative information derived from literary sources was an integral part in framing this work, choosing particular areas of focus, and analysis of quantitative data. The qualitative work will begin by addressing culture more broadly, as done in the introduction, and then move into definitions and descriptions of culturally competent practices. A complete and respected source of this information is The Purnell Model for Cultural Competence, which focuses almost entirely on the domestic, multicultural diversity aspect of cultural competence in United States healthcare. The first piece of information utilized from this source is the concise and holistic definition of "culture," which provides a launching point for this work and helps clear up the convoluted nature of the word. Some of the other theoretical ideas highlighted and drawn from this source are cultural self-awareness, cultural relativism, cultural imposition, cultural imperialism, and cultural leverage. These ideas are used to present a framework of cross cultural interaction, which can be applied to interactions between healthcare providers and patients. The actual Purnell Model for Cultural Competence was also distilled from the source and presented, so that an example model could be used as reference. Since there are a number of assumptions and concepts associated with this model, it was important that they be outlined in order to analyze what implementing culturally competent practices entail. This work also includes a figure from the source that provides a visual representation of the Purnell model and principles behind the different categories of classification. Finally, an example of the implementation of this model is presented, so that cultural evaluation in relation to patient treatment can also be analyzed. The qualitative information and theoretical framework outlined 
provide a context for the quantitative information drawn from other sources and generated for this work.

According to the Purnell Model for Cultural Competence cultural self-awareness is an important first step for healthcare providers to move outside their personal biases towards cross cultural understanding. This process of critical self-examination involves addressing personal and professional knowledge, attitudes, beliefs, values, ethics, and life experiences. Purnell argues that this process allows the healthcare provider to optimize their cultural assessment of the individual, since understanding of one's own existence assists in allowing them to neutralize their own biases when dealing with culturally diverse patients. This also raises the issue of cultural relativism, which is the idea that a culture can only truly be understood by individuals within it and judgment of any practices should not come from the outside world. This is an important point of departure, since this work looks precisely at healthcare providers not necessarily being members of the culture of the patients they treat. Also, considering that this work is looking at healthcare in the United States, cultural values or beliefs are only considered acceptable if they do not violate the state or federal laws, which then move this issue to one of cultural imposition.

Cultural imposition is the relentless application of the cultural values of the majority on all individuals or groups. This idea especially impacts the relationship between culture and healthcare because practices in the United States tend to be overarching. For instance, the practice of prescribing dietary changes for the treatment of certain illnesses is often made without consideration of the individual's cultural food choices, access, and options. This is similar, but not identical, to cultural imperialism, which is the dominant culture explicitly enforcing restrictive practices and policies on minority cultures. Although practices like these 
have long been made illegal in the United States through social rights activism, it is present in the inherent structure of the healthcare system. Healthcare in the United States is empirical in nature and relies on scientific principles. This often leads to healthcare providers minimizing and downplaying the significance or importance of other treatment methods that may be based in traditional cultural practices. The process of treating these different cultural biases and issues associated with cross-cultural understanding through implementing culturally competent practices is called cultural leverage. Cultural leverage is the process of facilitating behavioral change in the patient and provider, and this process for the Purnell Model is discussed next.

Understanding that cultural difference and biases exist, there still needs to be some general assumptions about culture made in order to have a model for evaluation and implementation. Purnell argues that for healthcare providers, it is safe to make some generalizations in order to facilitate proper consideration of a patient's culture. One key, and controversial, assumption is that there are inherent core values shared between all cultures. This is especially important because healthcare providers will always carry some level of cultural relativism; however establishing consistency in some core values allows a foundation for crosscultural understanding. Some other assumptions made are that cultures change slowly over time, there is variation between and among cultures, and culture has a powerful influence on interpretation and response to healthcare. What this information suggests is that there is a strong connection between cultural competence and the effectiveness of healthcare treatment, and these assumptions also provide the groundwork for the concepts of the Purnell Model. 


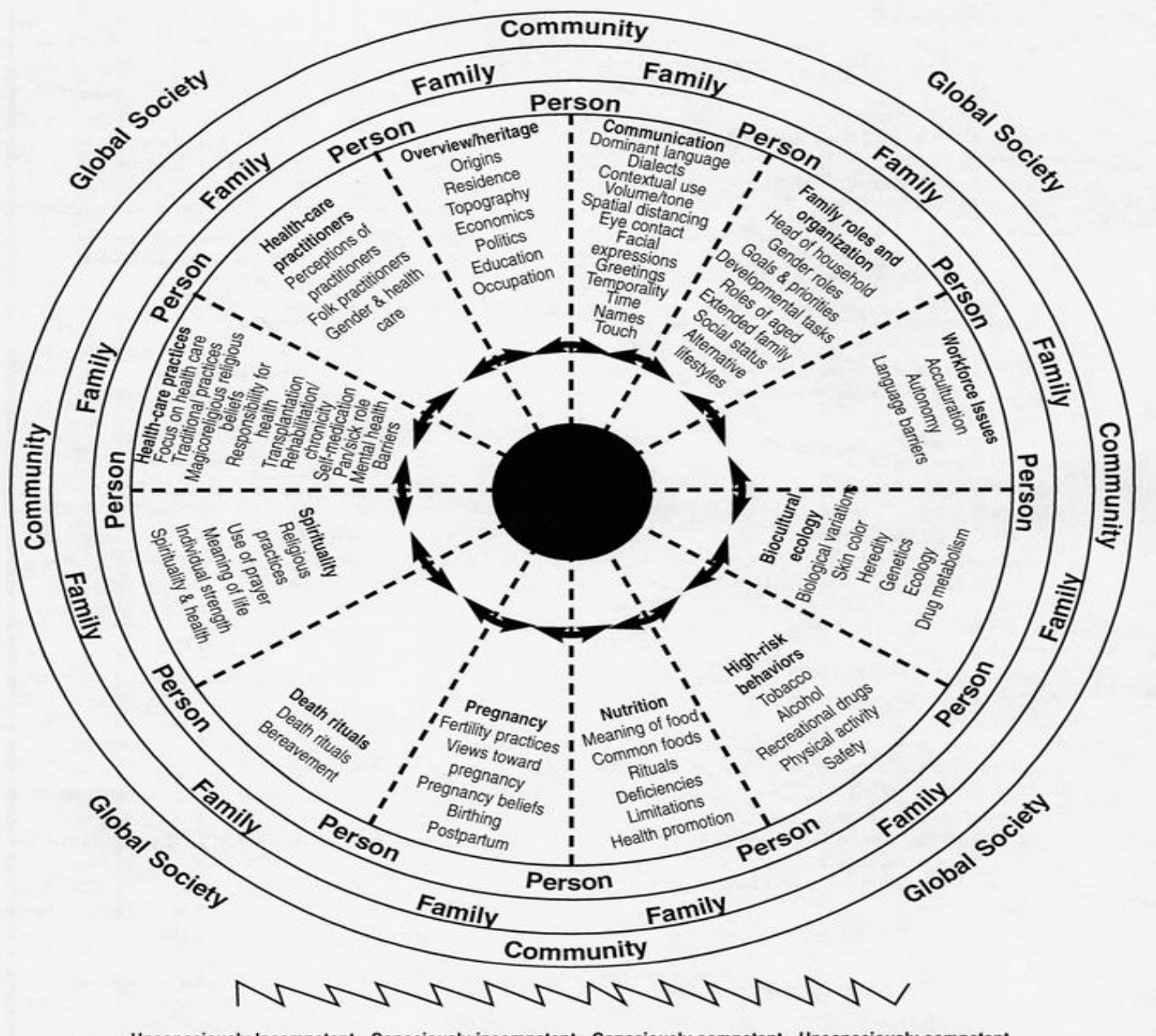

Unconsciously Incompetent - Consciously incompetent - Consciously competent - Unconsciously competent

Figure 1: The Purnell Model for Cultural Competence

The figure above is a visual representation of the Purnell Model. The outer rings of the circle represent what are described as "metaparadigms." They represent different levels of culture and society, beginning with the global society and moving to the level of the individual. The 12 interior wedges of the circle represent what Purnell calls the 12 cultural domains or constructs, as well as examples of the concepts that fit within them. The jagged line on the bottom represents the non-linear or direct path to achieving unconscious competency. What this model is intended to do, is provide an organizational framework for healthcare providers to 
access when considering the types of assessments that should be made for culturally diverse patients. One of the 12 domains this project was able to address was high-risk behaviors, namely substance abuse treatment in relation to patient diversity.

\section{Methods}

\section{Quantitative information generated from outside sources:}

The quantitative data utilized from outside sources was difficult to find, which highlights the necessity of this type of analysis. Little information exists pertaining to economic costs and benefits associated with culturally competent practices in healthcare settings. However, there was a source that looked into economic impacts of cultural competency in a variety of work environments. Although this source was not used significantly, it did contain data about the profit return for companies based on diversity understanding. It is important to stress that this data is not specific to hospitals and medical centers, but it provides a point of departure for discussing how cultural competence may have economic impacts on any institution, including healthcare.

Another type of quantitative information utilized from other sources is census data pertaining to demographic changes in the United States. As this work inspects the need for greater implementation of culturally competent practices, one major aspect to look into is whether cultural diversity is increasing in the United States. The first, and most obvious, source for demographic changes used for this work is the government census website, which contains reports on demographic changes between the decades when the information was collected. For this work, the change in data from 2000-2010 will be used, since it represents the most recent trends in population change for the United States. Another source of census data utilized was the 
Georgetown University site on cultural competence, which contains more specific data pertaining to changing demographics, such as trends in English speaking homes and changes with respect to children and race. This type of data was collected in order to analyze whether trends in population change in the United States suggest increasing cultural diversity. If a relationship between cultural competence and healthcare can be established, then the need for greater implementation due to growing diversity can be suggested.

Data pertaining to changing diversity in the U.S. population as a whole will then be compared to physician diversity. As outlined in the introduction, a major response to increasing cultural competency in the U.S. was an attempt to increase provider diversity. Two figures were taken from the AAMC Diversity in the Physician Workforce report for 2010. The data corresponds to physician diversity from 2008 , which can be compared to overall demographic changes of the same period. The first figure drawn from the report represents physician diversity in 2008 for ethnic groups that correspond nicely with those groups displayed in the census data. The second figure looks at the same groups, but compares the number of physicians graduating in each group from 1978-2008. These figures will allow for the analysis of current trends in physician diversity in the U.S., which can then be compared to the country as a whole. The quantitative data from other sources, along with the qualitative information, were compiled to use in conjuncture with healthcare data collected and generated for the purposes of analyzing patient cultural diversity with respect to cost and treatment.

\section{Quantitative information collected and generated:}

The charts created relate changes in Racial/ Ethnic Minority Treatment to changes in cost per patient, substance abuse treatment, and enabling services came from the U.S. Department of 
Health and Human Services Health Center Data and Statistics. The website contains health data from 2007-2012 reported by state, and totals for the entire United States. An interesting feature of the data is that it presents a trend for the percent change from 2010-2012. The major categories of data are: Age and Race/ Ethnicity, Patient Characteristics, Services, Clinical Data, and Cost Data. In order to understand whether treating culturally diverse patients has an effect on cost and services, I analyzed the state and territory records and compared them based on those who saw an increase or decrease in the percent change of Racial and/or Ethnic Minority patients treated. What this allowed for was analysis of the relationship between increasing or decreasing cultural diversity in patients and other factors presented in the data.

To begin, the individual state records presented by the Health Center Data and Statistics were accessed. Each state was first assessed and grouped according to whether there was a decrease or increase in the percent change in Racial and/ or Ethnic Minority patients treated from 2010-2012. Since this study was initially designed to analyze costs and benefits of culturally competent practices, it made sense to first look at the change in cost per patient for each state from 2010-2012. All of the states/ territories that saw an increase in minority patient treatment were grouped together, and the average change in patient cost for those locations was able to be determined. The same process took place with regards to those states/ territories that saw a decrease in minority patient treatment. The average change in cost per patient for those regions was calculated, which then allowed the two types groups to be compared on the basis of the change in cost per patient.

The data bank contains many different types of information for each state, so it was important to identify categories of data presented that may influence or be influenced by the relationship between patient culture and healthcare, if such a relationship exists. One aspect of 
healthcare services that the literature suggested may be worth addressing is the relationship between changing cultural diversity in patients and enabling services required. It may seem obvious to look into services specifically aimed at helping those with limited access to different healthcare services, but it is an important relationship for this analysis. The data for this was collected similarly as was done for the change in cost per patient. The change in enabling services reported from 2010-2012 for each region was recorded, and the average change for all regions that experienced an increase in Racial and/or Ethnic Minority patients treated was calculated. Then, the average change in enabling services for those regions that saw a decrease in minority patient treatment was calculated, so that the two groups differing with regards to change in minority treatment could be compared based on the change in enabling services they needed to provide.

The final category of information drawn from the data bank was a specific example of a possible influence culture may have on patient treatment. It is a common notion that cultural minority groups, especially in the United States, tend to have issues with substance abuse. If there is data to support or refute this premise, then perhaps treatment and preparation can be more specifically designed or targeted for groups that have greater amounts of diversity. The steps for collecting the data was almost identical to those stated previously. The change in substance abuse treatment from 2010-2012 was collected for each state/ territory and grouped according to whether it corresponded to a region that experienced an increase or decrease in Racial and/or Ethnic Minority patients treated. The average change in substance abuse treatment for increasing and decreasing minority patients were then determined and able to be compared to one another. 


\section{Results/ Findings}

One example of the Purnell Model being practically implemented is a case in a rural Delaware community where the healthcare department integrated the model in response to the influx of immigrants, especially Haitians. They report an estimated $29 \%$ increase in minority populations in United States nonmetropolitan areas in the last decade (Johnson and Phelps, 2004, p. 203). This county saw a drastic increase in the Haitian population, which grew to about $3 \%$ of the total population. There are several cultural barriers that influenced Haitian patient treatment in this county, such as poor communication due to language barriers and perceptions of Haitian culture being associated with voodoo and HIV/AIDS. In response, the county health department implemented the Purnell Model by creating a website for Haitians to address healthcare practices in relation to their cultural background. The results of the program showed that the education and tools compiled allowed providers to practice more culturally sensitive healthcare, which led to greater patient satisfaction and receptiveness of treatment.

In The Impact of Cultural Competency/ Diversity, Samuel Hancock analyzes cultural competency and evaluation with the lens being more focused on business and economic outcomes. Although most of the information is not exclusive to healthcare, which is the lens of this project, the findings from Hancock's work were a driving force for the formulation of this thesis. 


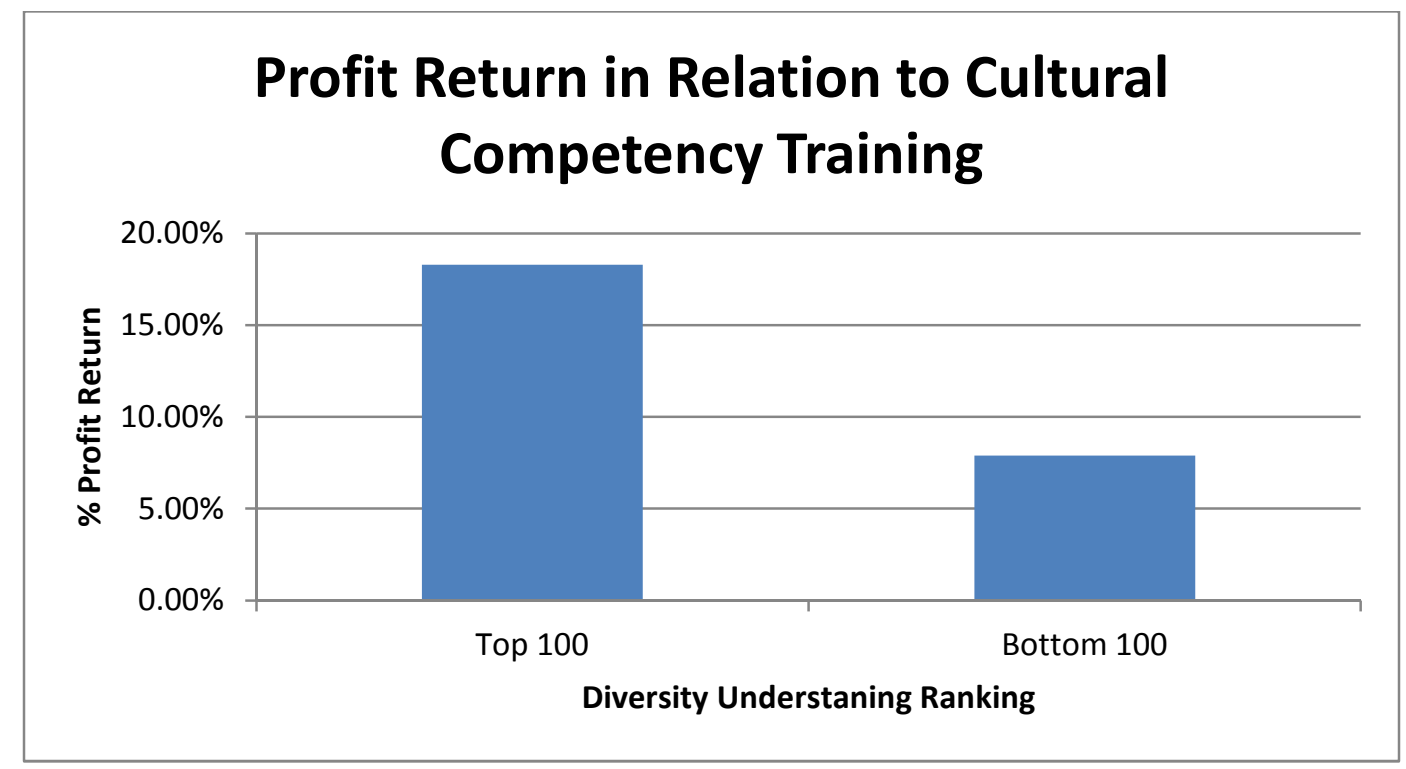

Figure 2: A comparison of the percent profit return for S\&P 500 companies ranked in the top or bottom 100 in cultural diversity understanding

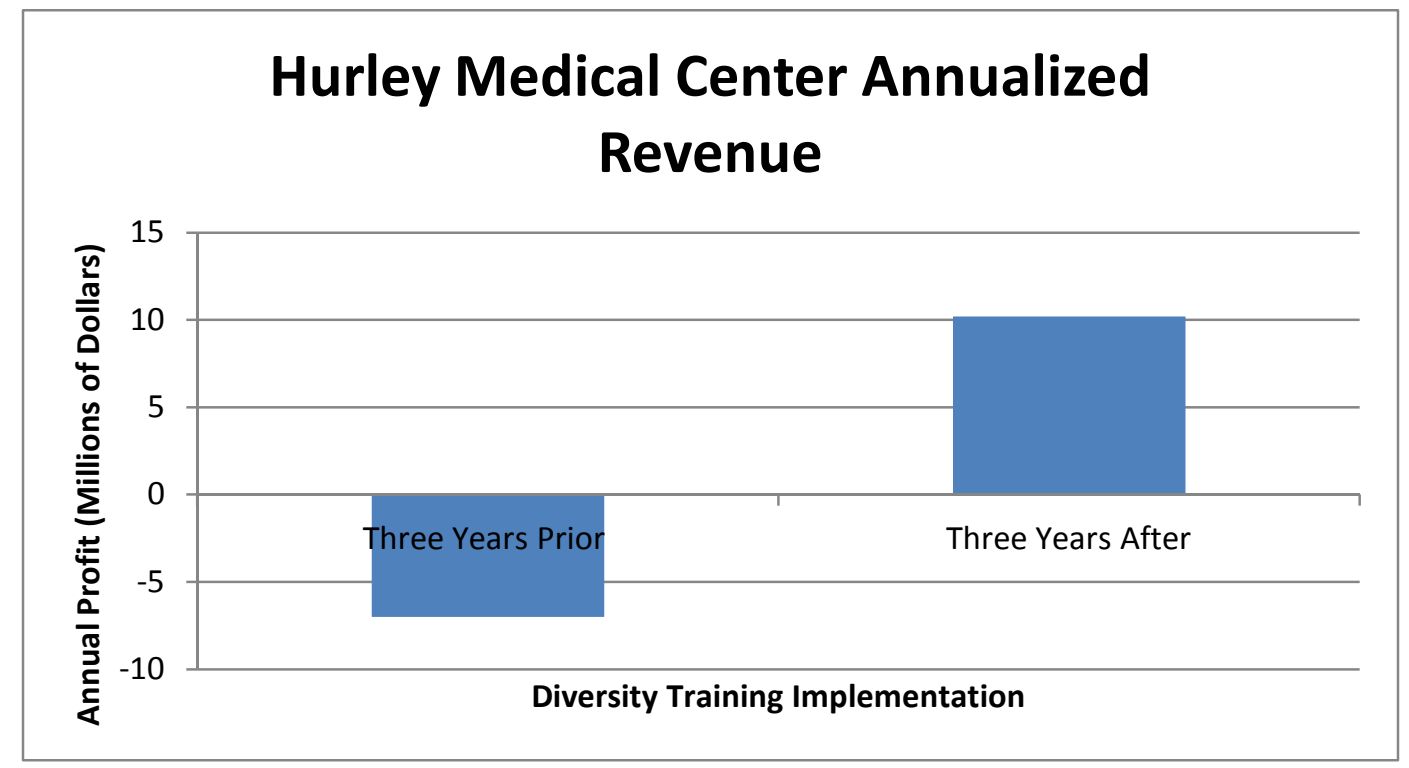

Figure 3: Revenue for Hurley Medical Center in Michigan before and after implementing cultural diversity training

Figures 2 and 3 displayed above were created from information drawn from Hancock's analysis on cultural diversity training. Figure 2 shows an analysis of S\&P 500 companies, and their profit return in relation to their diversity training ranking. Of those ranked in the top 100 a 
profit return of $18.3 \%$ was experienced, compared to just $7.9 \%$ for those in the bottom 100 . Although this takes an array of businesses and diversity of institutions into account, it clearly indicates profitability may be related to cultural competency. When this is considered with the analysis of Hurley Medical Center, which experienced a $\$ 10.2$ million annual profit for the three years following implementing cultural competency training after a $\$ 7.2$ million annual deficit the three years prior, it becomes evident that the relationship between profitability and cultural competency may be especially prevalent in hospitals and medical centers. Note that the $\$ 10.2$ million profit was the largest experienced by the institution in the last 10 years. The next piece of information drawn on to analyze the need for culturally competent practices in the U.S. is census data pertaining to racial/ cultural demographic changes.

Before getting into changing U.S. demographics, it is important to note that cultural demographics are not being treated to the full extent possible. Of course when discussing culture, there are a multitude of individuals who identify with multiple groups, however those individuals are not addressed for the purposes of this work. Census data corresponding to changes from 1990-2000, will first be briefly discussed as Georgetown University synthesized findings that yield interesting insight. The census data most pertinent to this work is looking into more general trends in cultural diversity in the U.S. from 2000-2010; in order to establish the need for greater implementation of culturally competent practices.

According to census data from 1990-2000 utilized by Georgetown University, the number of non-English speaking homes increased by 28.3 million, which represents a $43 \%$ rise. They also reported that the U.S. foreign born population comprised $10 \%$ of the total population; a larger segment than any time in the last 50 years. Changing demographics with respect to U.S. children for this decade is also presented, and may feed into the trends for the subsequent decade. 
From 1990-2000, the number of Hispanic/ Latino children increased by 5.5 million, African American children increased by 2.6 million, children of other races increased by 1.5 million, while the number of Caucasian children decreased by 6.2 million. This data is important to consider along with demographic information for the U.S. for the most recent decade, as well as data pertaining to the demographics of healthcare providers.

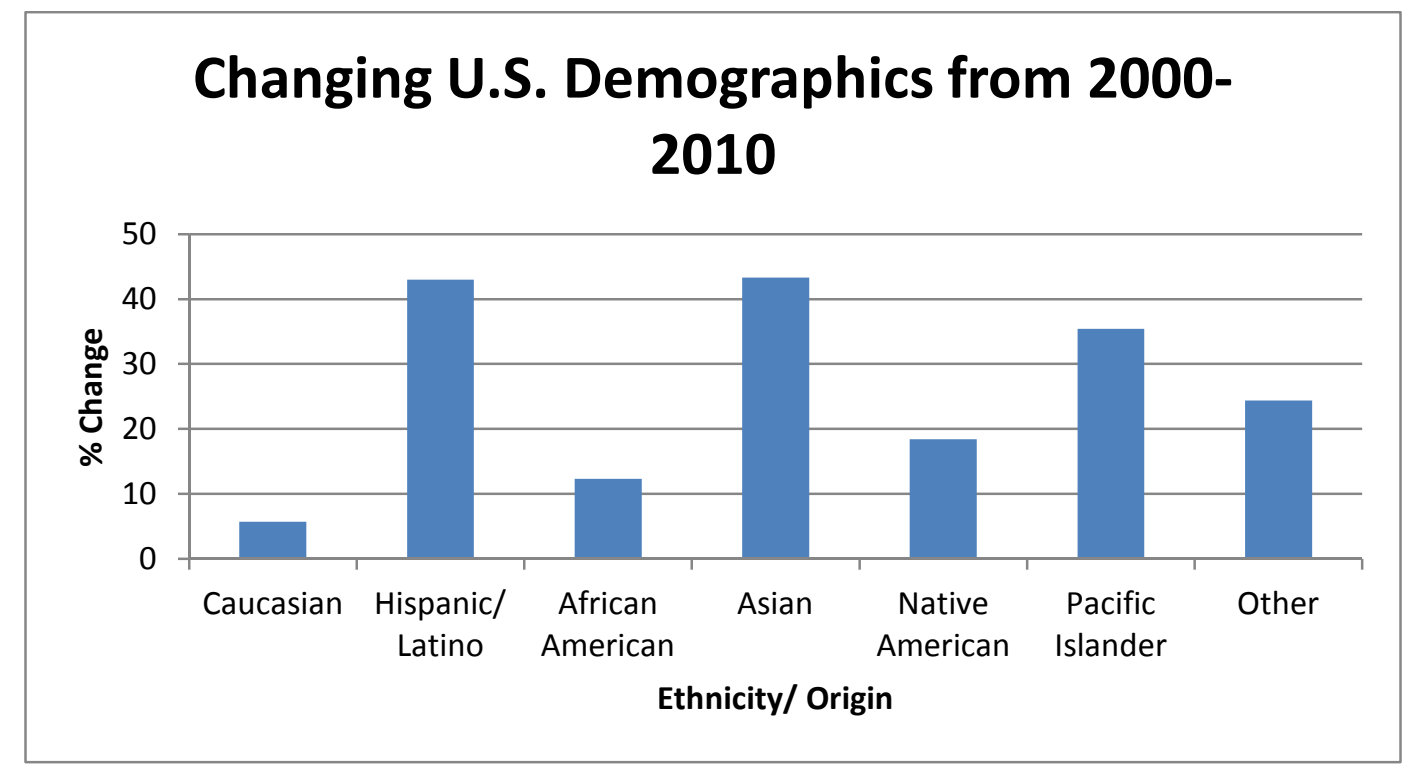

Figure 4: Demographic changes drawn from U.S. Census data, 2000-2010

The figure above was created using data from the U.S. census, and represents demographic changes from 2000-2010. Note that just as the number of Caucasian children in the U.S. actually saw a decrease from 1990-2000, the Caucasian population from 2000-2010 saw the lowest increase in population as compared to the other ethnic groups presented. The more significant and drastic increases in the Hispanic/ Latino, Asian, and Pacific Islander groups may suggest that cultural competency and evaluation in the U.S. should be geared more toward those groups. However what is most important to take from this data, is that the changing demographics in the U.S. suggest increasing cultural diversity in the population as a whole, which in turn creates a need for more culturally competent healthcare practices. As I outlined in 
my introduction, one of the responses in an attempt to improve cultural competency was to push for increased numbers of providers who are representative of cultural minority groups.

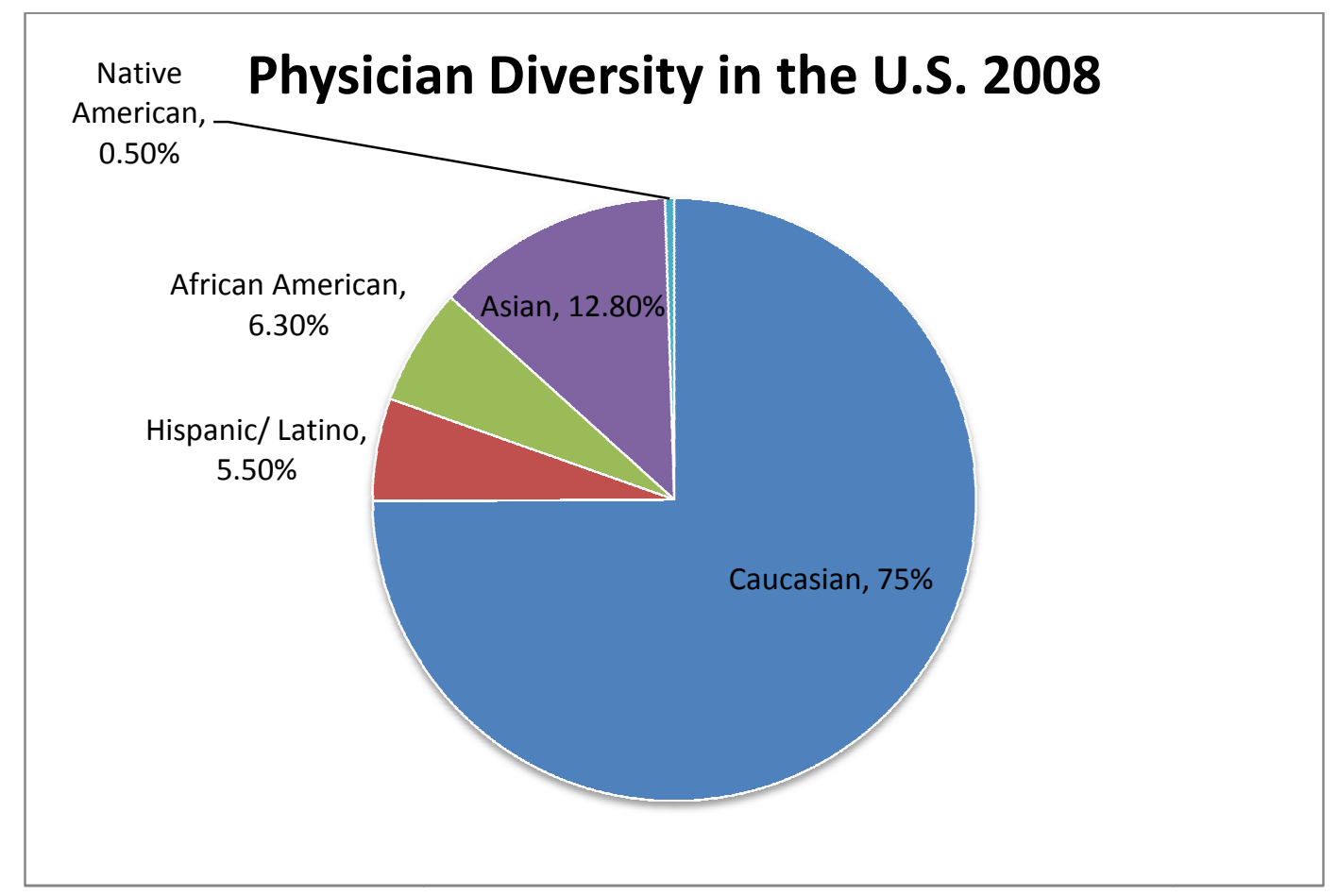

Figure 5: U.S. Physician Diversity from 2010 report

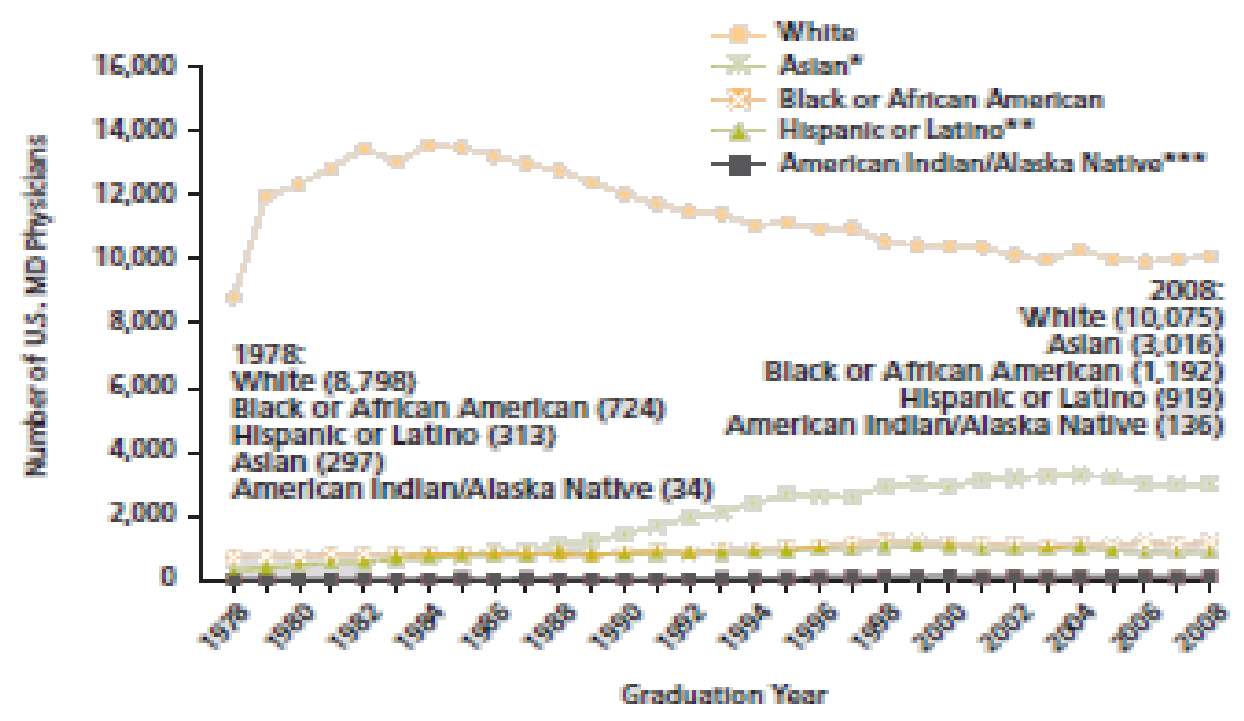

Figure 6: Changes in Physician Graduation based on Ethnicity (Castillo-Page, 2010) 
Figures 5 and 6 were taken from the AAMC report, Diversity in the Physician Workforce. The most immediately apparent insight is from Figure 5, which shows that Caucasian physicians comprised $3 / 4$ of the entire physician workforce in the U.S. in 2008 . The current physician workforce seems to lack the cultural diversity representative of the population, and recent trends suggest that this is not likely to change significantly. Although Figure 6 shows that diversity in the physician workforce has increased to some extent since 1978, after the mid 1990's the number of physicians who are representative of the ethnic groups presented remains relatively static, with Caucasian/ White maintaining a significant majority. As shown by the previous census data, diversity in the U.S. as a whole has been increasing drastically since at least 1990, yet this data suggests that diversity in the physician workforce has remained constant since that time. Clearly the hopes of increasing physician diversity in the U.S. in order to improve cultural competency has gone largely unfulfilled.

Drawing upon the individual state data from the U.S. Department of Health and Human Services, I was able to generate a figure to compare healthcare treatment with respect to increasing and decreasing ethnic diversity from 2010-2012. The figure below shows the average percent change in cost per patient, enabling services, and substance abuse treatment in order to gain insight into how changing cultural demographics affect healthcare. The average change in cost per patient for those state hospitals and medical centers experiencing greater ethnic diversity was an increase of $10.7 \%$, and it was only a $7.1 \%$ increase in cost for those experiencing a decrease in ethnic diversity. The most significant difference is with respect to average change in enabling services, which went up $21.3 \%$ with increasing ethnic diversity and only $5.1 \%$ with decreasing diversity. Interestingly, the average change in substance abuse treatment went down $3.8 \%$ with increasing diversity and up $9.7 \%$ with decreasing diversity. 


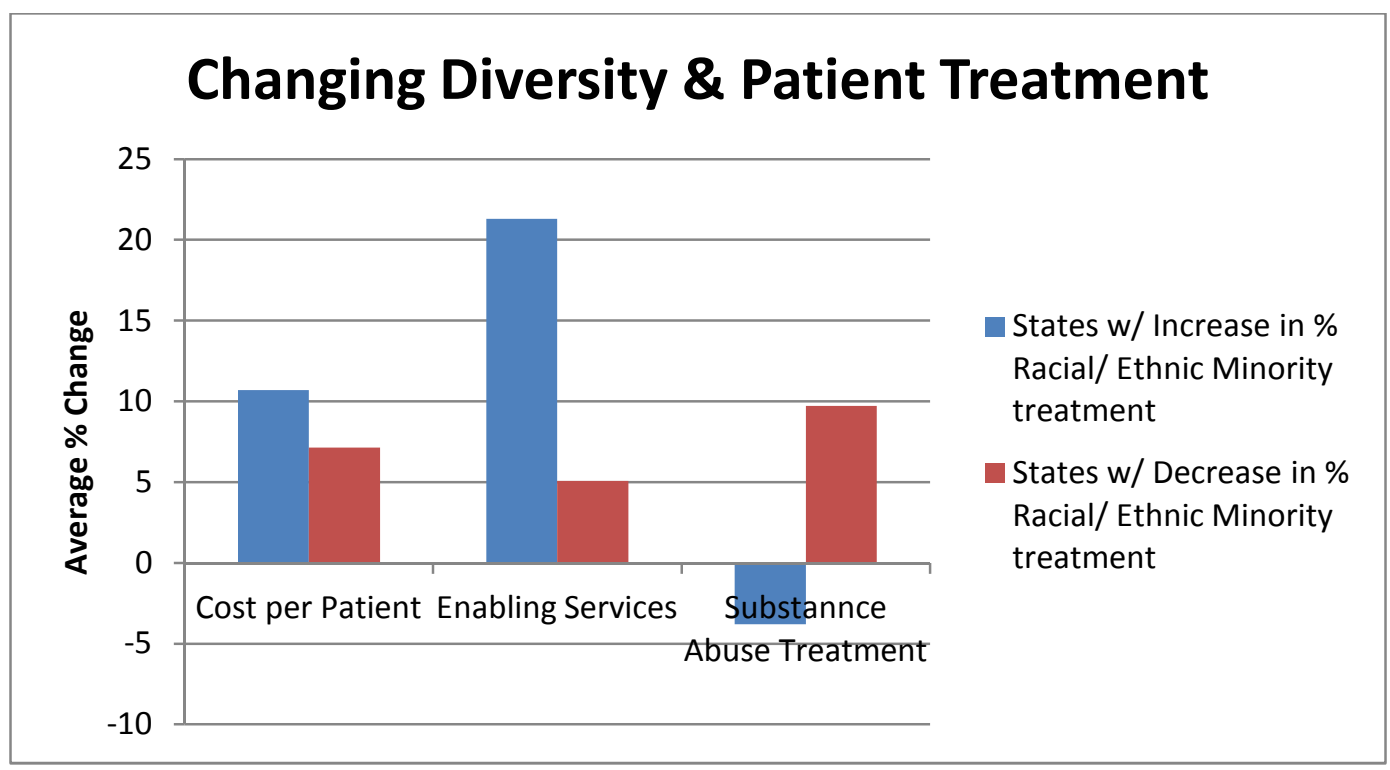

Figure 7: Changes in patient services for states experiencing an increase or decrease in patient diversity from 2010-2012.

\section{Discussion/ Conclusions}

As far as the entire country is concerned, the U.S. Census data showed that overall ethnic/ cultural diversity is on the rise. Interestingly, even with this information and the supposed response to compensate demographic changes with increased physician diversity the data from AMCAS does not support that is taking place. Even though the Caucasian/ White group represents the smallest increase in population from 2000-2010, in 2008 they represented $75 \%$ of the physician workforce. This would not be as big of an issue if trends suggested that some changes are occurring. Yet when we look at physician diversity trends since 1978, the only significant changes occurred in the late 1980's and early 90's. Even these changes were not drastic enough to represent a deep structural change to the way healthcare is provided. In the U.S. the physician workforce has and continues to be dominated by Caucasian/ White individuals even in the face of constantly increasing diversity of the population in the country. 
This is perhaps the greatest opportunity to influence culturally competent healthcare, as increasing physician diversity could help alleviate some of the cross-cultural barriers to understanding. However, this is not a realistic endeavor as there are socioeconomic factors related to becoming a physician, and affirmative action policies tend to fuel ideas of difference. As was seen with the Haitian patient treatment in rural Delaware, the Purnell Model may be an effective starting point for other hospitals and medical centers to establish competency and evaluation practices.

The data compiled and generated from the U.S. Department of Health and Human Services does suggest a need to attend to culture in healthcare. The data suggests that increasing diversity results in greater cost per patient, which perhaps correlates with the fact that the care is being handled inadequately and inefficiently. With greater patient diversity, there may be a disconnect between the patient and physician inhibiting effective communication of treatment options and receptiveness of the treatment itself. Those factors inevitably influence how cost effective the patient treatment will be. This data is synonymous with the economic data drawn from Hancock's work looking at costs and benefits of culturally competent practices. Together, the implications are that culturally competent practices have real financial effects on the institutions and the individuals receiving treatment. Implementing or improving culturally competent practices may be an effective avenue for hospitals and medical centers seeking to improve their own annual profit returns while reducing the cost of treatment per patient.

The changes in enabling services represent the most significant difference with respect to changing patient diversity. Enabling services are inherently tied to ethnically or culturally diverse patients, since they encompass areas like linguistic services to improve access to healthcare. Although it might seem obvious that increasing diversity resulted in an increased 
need for enabling services, an empirical connection between them in hospitals and medical centers was previously unknown. For hospitals and medical centers in areas of the U.S. experiencing increases in patient diversity, improved enabling services are a proactive way of addressing cultural competency without needing to implement major cultural evaluation programs. This will in turn influence costs for the patient and institution by allowing effective communication of treatment options and strategies. As the demographics showed, diversity in the U.S. as a whole has been increasing dramatically, so it is vital that enabling services are improved and increased across all U.S. hospitals and medical centers.

Taking on the particular focus of one of the 12 cultural domains outlined by Purnell's Model of cultural competence provides a specific lens through which culturally competent practices can be addressed at the patient level. Substance abuse associated with "high-risk behavior" is typically perceived to be a more significant issue within cultural minority groups. However, as the data shows there was actually a decrease in the average percent change in substance abuse treatment for those state hospitals and medical centers experiencing an increase in patient diversity. What this shows is that culturally competent practices cannot be geared toward improving services that are perceived to be more substantial to a particular group. Each of the 12 cultural domains needs to be empirically assessed specifically to a particular group, ideally in a particular state or even city, in order to understand how patient services need to be adjusted. It is not adequate to assume substance abuse is a greater issue for culturally diverse groups compared to Caucasian/ White patients because as this data shows, the perception is not consistent with reality. In order to improve cultural competency and all of the factors outlined that surround it, this sort of evaluation needs to be done to know whether a particular institution requires improvements in services that will improve cultural understanding of the prevalent 
cultural groups. What should be drawn from this work is that cultural competency has tangible impacts on patient treatment, and my hope is that it will emphasize the need for the implementation of training or development programs that will formally instruct health professionals on cultural evaluation.

Some of the possible avenues future work may take include: actual economic costs/benefits pertaining to the business aspects of cultural competency, globalization and whether the growing interconnected world community requires more or less cross-cultural understanding, and how scientific discourse can be integrated into real world subjective interactions so the general patient and specific individual can both be accounted for. Although this work was able to address possible economic aspects related to cultural competency, truly understanding the influences and implications requires systematic studies of numerous institutions' financial records in conjunction with records on patient culture. One issue that is interesting to consider is the effect of globalization on cultural diversity. Will there ever be a time when the world's cultures are completely homogenized? Although this seems extreme and unlikely, the implications of an interconnected world may have significant impacts on culture and healthcare in the future. The final point to consider is perhaps the most integral to this work, and the most pivotal next step. Healthcare is a scientific endeavor that revolves around the study of people, which are complex social and cultural creatures. At its core this work has attempted, in both structure and content, to show how the gaps between science and society may be bridged. Scientific discourse is obsessed with the empirical and objective, but the world is not a laboratory of defined variables. In order to have science optimally function for society, there has to be some level of integration of subjective human experience with scientific principles. 


\section{References}

APHA. 2013. “Advocacy \& Policy.” American Public Health Association.http://www.apha.org.

Beach, MC, EG Price, and TL Gary, et al. 2005. "Cultural Competency: A Systematic Review of Health Care Provider Educational Interventions." Med Care 43 (4) (April): 356-373. http://www.ncbi.nlm.nih.gov/pmc/articles/PMC3137284/.

Castillo-Page, Laura. 2010. "Diversity in the Physician Workforce Facts \& Figures 2010”. Association of American Medical Colleges. https://members.aamc.org/eweb/upload/Diversity\%20in\%20the\%20Physician\%20Workf orce $\% 20$ Facts $\% 20$ and $\% 20$ Figures $\% 202010 . p d f$

Georgetown University. "The Compelling Need for Cultural and Linguistic Competence." National Center for Cultural Competence. http://nccc.georgetown.edu/foundations/need.html.

Hancock, Samuel. 2005. "The Impact of Cultural Competency/Diversity.” Dissertation, Ohio: Bowling Green State. http://www.utoledo.edu/offices/oid/pdf/hancock_jan10_05.pdf.

Hopson, Rodney. 2003. "Multicultural Health Evaluation”. The California Endowment.http://www.calendow.org/uploadedFiles/Publications/Evaluation/Multicultur al_Health_Evaluation/OverviewBook.pdf.

Humes, Karen, Nicholas Jones, and Roberto Ramirez. 2011. “Overview of Race and Hispanic Origin: 2010." March. http://www.census.gov/prod/cen2010/briefs/c2010br-02.pdf.

Kleinman, Arthur, and Peter Benson. 2006. "Anthropology in the Clinic: The Problem of Cultural Competency and How to Fix It.” PLoS Medicine 3 (10) (October24).http://www.plosmedicine.org/article/info\%3Adoi\%2F10.1371\%2Fjournal.p med.0030294.

Kodjo, Cheryl. 2009. "Cultural Competence in Clinician Communication.” National Institutes of Health 30 (2) (February): 57-64. http://www.ncbi.nlm.nih.gov/pmc/articles/PMC2719963/. 
Nordqvist, Christian. 2012. “A History of Medicine.” Medical News Today. August. http://www.medicalnewstoday.com/info/medicine/.

Phelps, Lisl, and Karin Johnson. 2004. "Developing Local Public Health Capacity in Cultural Competency: A Case Study with Haitians in a Rural Community." Journal of Community Health Nursing 21 (4) (Winter): 203-215. http://www.jstor.org/stable/3427827?seq=1\&.

Purnell, Larry. 2011. “The Purnell Model for Cultural Competence.” In Intervention in Mental Health-Substance Use, 29-50. RadcliffePublishing.

U.S. Department of Health and Human Services. "Primary Care: The Health Center Program." Health Resources and Services Administration. http://bphc.hrsa.gov/index.html. 